\title{
ALMA observations of lensed Herschel sources: testing the dark matter halo paradigm
}

\author{
A. Amvrosiadis, ${ }^{1 \star}$ S. A. Eales, ${ }^{1}$ M. Negrello, ${ }^{1}$ L. Marchetti,,${ }^{2,3,4}$ M. W. L. Smith, ${ }^{1}$ \\ N. Bourne,${ }^{5}$ D. L. Clements, ${ }^{6}$ G. De Zotti, ${ }^{7}$ L. Dunne, ${ }^{1}$ S. Dye,${ }^{8}$ C. Furlanetto, ${ }^{1}$ \\ R. J. Ivison, ${ }^{5,9}$ S. J. Maddox, ${ }^{1}$ E. Valiante, ${ }^{1}$ M. Baes,${ }^{10}$ A. J. Baker,${ }^{11}$ A. Cooray, ${ }^{12}$ \\ S. M. Crawford, ${ }^{4}$ D. Frayer, ${ }^{13}$ A. Harris,${ }^{14}$ M. J. Michałowski, ${ }^{5,15}$ H. Nayyeri, ${ }^{12}$ \\ S. Oliver, ${ }^{16}$ D. A. Riechers, ${ }^{17}$ S. Serjeant ${ }^{3}$ and M. Vaccari ${ }^{18,19}$ \\ ${ }^{1}$ School of Physics and Astronomy, Cardiff University, The Parade, Cardiff CF24 3AA, UK \\ ${ }^{2}$ Department of Physics and Astronomy, University of the Western Cape, Private Bag X17, 7535 Bellville, Cape Town, South Africa \\ ${ }^{3}$ School of Physical Sciences, The Open University, Walton Hall, Milton Keynes MK7 6AA, UK \\ ${ }^{4}$ South African Astronomical Observatory, PO Box 9, Observatory, 7935 Cape Town, South Africa \\ ${ }^{5}$ Institute for Astronomy, University of Edinburgh, Royal Observatory, Edinburgh EH9 3HJ, UK \\ ${ }^{6}$ Astrophysics Group, Imperial College, Blackett Laboratory, Prince Consort Road, London SW7 2AZ, UK \\ ${ }^{7}$ INAF - Osservatorio Astronomico di Padova, Vicolo dell Osservatorio 5, I-35122 Padova, Italy \\ ${ }^{8}$ School of Physics and Astronomy, University of Nottingham, University Park, Nottingham NG7 2RD, UK \\ ${ }^{9}$ Department of Physical Science, The Open University, Milton Keynes MK7 6AA, UK \\ ${ }^{10}$ Sterrenkundig Observatorium, Universiteit Gent, Krijgslaan 281 S9, B-9000 Gent, Belgium \\ ${ }^{11}$ Department of Physics and Astronomy, Rutgers, the State University of New Jersey, 136 Frelinghuysen Road, Piscataway, NJ 08854-8019, USA \\ ${ }^{12}$ Department of Physics and Astronomy, University of California, Irvine, CA 92697, USA \\ ${ }^{13}$ National Radio Astronomy Observatory, Green Bank, WV 24944, USA \\ ${ }^{14}$ Department of Astronomy, University of Maryland, College Park, MD 20742, USA \\ ${ }^{15}$ Astronomical Observatory Institute, Faculty of Physics, Adam Mickiewicz University, ul. Stoneczna 36, PL-60-286 Poznań, Poland \\ ${ }^{16}$ Department of Physics and Astronomy, University of Sussex, Brighton BN1 9QH, UK \\ ${ }^{17}$ Department of Astronomy, Cornell University, Ithaca, NY 14853, USA \\ ${ }^{18}$ Department of Physics and Astronomy, University of the Western Cape, Robert Sobukwe Road, 7535 Bellville, Cape Town, South Africa \\ ${ }^{19}$ INAF - Istituto di Radioastronomia, via Gobetti 101, I-40129 Bologna, Italy
}

\begin{abstract}
With the advent of wide-area submillimetre surveys, a large number of high-redshift gravitationally lensed dusty star-forming galaxies have been revealed. Because of the simplicity of the selection criteria for candidate lensed sources in such surveys, identified as those with $S_{500 \mu \mathrm{m}}>100 \mathrm{mJy}$, uncertainties associated with the modelling of the selection function are expunged. The combination of these attributes makes submillimetre surveys ideal for the study of strong lens statistics. We carried out a pilot study of the lensing statistics of submillimetreselected sources by making observations with the Atacama Large Millimeter Array (ALMA) of a sample of strongly lensed sources selected from surveys carried out with the Herschel Space Observatory. We attempted to reproduce the distribution of image separations for the lensed sources using a halo mass function taken from a numerical simulation that contains both dark matter and baryons. We used three different density distributions, one based on analytical fits to the haloes formed in the EAGLE simulation and two density distributions [Singular Isothermal Sphere (SIS) and SISSA] that have been used before in lensing studies. We found that we could reproduce the observed distribution with all three density distributions, as long as we imposed an upper mass transition of $\sim 10^{13} \mathrm{M} \odot$ for the SIS and SISSA models, above which we assumed that the density distribution could be represented by a Navarro-FrenkWhite profile. We show that we would need a sample of $\sim 500$ lensed sources to distinguish between the density distributions, which is practical given the predicted number of lensed sources in the Herschel surveys.
\end{abstract}

Key words: gravitational lensing: strong-galaxies: high-redshift-submillimetre: galaxies. 


\section{INTRODUCTION}

Photons travelling from a distant background source and through the vicinity of massive objects, such as galaxies or groups/clusters of galaxies, get deflected by the presence of their gravitational field. If the background source and the foreground object are well aligned with the observer, we have the creation of multiple images. This effect is called strong gravitational lensing (Schneider, Ehlers \& Falco 1992).

For a sample of these lensed sources, the statistics of angular separations mainly depends on four factors: (a) the luminosity function of the source population (More et al. 2012); (b) the number density of dark matter haloes as a function of halo mass and redshift (Eales 2015); (c) the mass density distributions within the haloes (Kochanek \& White 2001; Takahashi \& Chiba 2001; Oguri 2002, 2006); and (d) the cosmological model (Li \& Ostriker 2002, 2003; Chae 2003; Oguri et al. 2008, 2012). In principle, therefore, the statistics of image separations for a suitable sample of lensed sources is a powerful way of examining the mass density distribution of the total matter in the halo and halo mass functions predicted by simulations.

The two alternative methods for producing samples of strong lenses for statistical purposes are to start from either a population of objects that potentially act as lenses or from a population of potentially lensed sources. Follow-up observations are necessary in both cases to confirm the strong lensing nature. Examples of the first method are the Sloan Lens ACS (SLACS) Survey (Bolton et al. 2006) and the Baryon Oscillation Spectroscopic Survey (BOSS) Emission-Line Lens Survey (BELLS; Brownstein et al. 2012), in both of which the potential lensed systems were found by looking for galaxies with a spectrum that show two redshifts - with confirmation of the lensing provided by imaging with the Hubble Space Telescope. For our purpose of investigating the properties of haloes, the disadvantage of this approach is that it is prone to selection effects.

Examples of the second method were the Cosmic Lens All-Sky Survey (CLASS; Browne et al. 2003; Myers et al. 2003) and the Sloan Digital Sky Surveys Quasar Lens Search (SQLS; Oguri et al. 2006). CLASS was the largest survey of strongly lensed quasars conducted at radio wavelengths. Starting from a well-defined statistical sample of $\sim 9000$ flat-spectrum radio sources, the CLASS team used high-resolution radio observations to produce a statistically well-defined sample of 13 lensed sources (Browne et al. 2003). The SQLS selected potential lens candidates from the Sloan Digital Sky Survey (Oguri et al. 2006), producing a final catalogue (Inada et al. 2012) of 26 lensed quasars from an initial catalogue of $\sim 50000$ quasars. It is worth pointing out that both optical and radio surveys require huge parent samples in order to identify a few strong lenses.

With the advent of wide-area extragalactic surveys undertaken with Herschel Space Observatory (Pilbratt et al. 2010) at submillimetre wavelengths on the other hand, a new method for discovering high-redshift gravitationally lensed dusty star-forming galaxies has been made possible with an almost 100 percent efficiency. The number counts of unlensed submillimetre galaxies (SMGs) are very steep at bright flux densities (Blain 1996; Negrello et al. 2007). Therefore, the brightest sources after removing nearby galaxies and radio-loud active galactic nuclei (AGN) can be selected as candidate lensed sources, since there are very few high-redshift galaxies that are intrinsically so bright (effectively exploiting an extreme case of the magnification bias). Negrello et al. (2010) demonstrated this method for the first time, using the initial results from the Herschel Astrophysical Terahertz Large Area Survey (H-ATLAS; Eales et al. 2010). They showed that out of 10 extragalactic sources with flux $S>100 \mathrm{mJy}$ at $500 \mu \mathrm{m}$, five were strongly lensed high-redshift galaxies, with the remainder being easily identified as local $(z<0.1)$ spiral galaxies and in one case a previously known radio-bright AGN. Exploiting the whole $\sim 600 \mathrm{deg}^{2}$ area covered by H-ATLAS, Negrello et al. (2017) have identified a sample of 80 candidate strongly lensed SMGs using the same selection criteria. Follow-up observations with submillimetre interferometers or with the Hubble Space Telescope and W. M. Keck Observatory have confirmed so far that 20 of these extragalactic sources show a strong lensing morphology. Samples of lensed sources have now been produced using the same method from other Herschel surveys. A sample of 13 candidate strongly lensed galaxies was produced by Wardlow et al. (2013) from $95 \mathrm{deg}^{2}$ of the Herschel Multi-tiered Extragalactic Survey (HerMES; Oliver et al. 2012), 11 of which have been confirmed by follow-up observations to be strongly lensed. More recently, Nayyeri et al. (2016) produced a list of 77 candidate gravitationally lensed galaxies from the HerMES Large Mode Survey (HeLMS; Oliver et al. 2012) and the Herschel Stripe 82 Survey (HerS; Viero et al. 2014), which in total cover an area of $372 \mathrm{deg}^{2}$.

This uniform and simple selection technique that identifies potential candidates based on the emission from the source rather than the lens and so falls in the second category of methods. One of the main advantages of this technique is that submillimetre emission from the lens is usually negligible compared with the emission from the source. Therefore, the modelling of the lensed source emission in high-resolution submillimetre/millimetre imaging data does not suffer from uncertainties caused by the lens subtraction (Dye et al. 2014; Negrello et al. 2014).

Bussmann et al. (2013, hereafter B13) presented Submillimeter Array (SMA) $880 \mu \mathrm{m}$ observations of a sample of 30 candidates strong gravitational lenses identified from the two widest Herschel extragalactic surveys, H-ATLAS and HerMES. In our previous paper (Eales 2015) we investigated whether the standard dark matter halo paradigm could explain the distribution of Einstein radii measured from the SMA observations. We tried three halo mass functions, all estimated from numerical simulations that only included dark matter, and two different methods for calculating the lensing magnification produced by each dark matter halo. In all cases we found that the model predicted a larger number of sources with large Einstein radii than we observed. In this paper, we have extended and improved our previous study in several ways. First, the SMA results we used in our previous paper had limited angular resolution and sensitivity, and we were concerned that we might have missed arcs of large angular size with low surface brightness, causing us to underestimate the number of sources with large image separations. For this reason, we started a project to map the lensed Herschel sources with Atacama Large Millimeter Array (ALMA), and in this paper we present the first results from this ALMA project. We compare the distributions of image separations measured from the ALMA images with the predictions of our models. Our second improvement is to use a halo mass function and density distributions from the haloes derived from a numerical simulation that includes baryons and dark matter.

The layout of this paper is as follows. In Section 2, we present the first results from our ALMA project. In Section 3 we describe the halo models and lay down the theoretical background for computing the lensing properties of the haloes. Section 4 describes the comparison between the observed and predicted Einstein radii. We discuss our results in Section 5. Throughout this paper, we assume a 
Table 1. The ALMA sample.

\begin{tabular}{|c|c|c|c|c|c|c|}
\hline IAU name & $\begin{array}{l}\text { Other } \\
\text { name }\end{array}$ & $\begin{array}{c}500-\mu \mathrm{m} \text { flux } \\
\text { density (mJy) }\end{array}$ & $z_{1}$ & $z_{\mathrm{s}}$ & $\theta_{\mathrm{E}}(\operatorname{arcsec})$ & Ref. \\
\hline HeLMS J001615.8+032435 & HeLMS13 & $149 \pm 7$ & 0.663 & 2.765 & $5.22 \pm 0.05$ & N16 \\
\hline HeLMS J001626.2+042612 & HeLMS22 & $127 \pm 7$ & 0.2154 & 2.509 & $0.98 \pm 0.05$ & M17, N16 \\
\hline HeLMS J004714.2+032453 & HeLMS8 & $168 \pm 8$ & 0.478 & 1.195 & $0.58 \pm 0.05$ & N16 \\
\hline HeLMS J004723.5+015750 & HeLMS9 & $164 \pm 8$ & 0.3650 & 1.441 & $2.66 \pm 0.05$ & M17, N16 \\
\hline HeLMS J005159.4+062240 & HeLMS18 & $135 \pm 7$ & - & 2.392 & $6.54 \pm 0.05$ & N16 \\
\hline H-ATLAS J083051.0+013225 & G09v1.97 & $269 \pm 9$ & 0.626 & 3.634 & $0.85 \pm 0.04$ & B13, MN17 \\
\hline H-ATLAS J083344.9+000109 & - & $96 \pm 9$ & - & 2.530 & - & M17 \\
\hline H-ATLAS J085358.9+015537 & G09v1.40 & $228 \pm 9$ & - & 2.089 & $0.55 \pm 0.04$ & B13, MN16, M17 \\
\hline H-ATLAS J141351.9-000026 & G15v2.235 & $176 \pm 9$ & 0.547 & 2.478 & - & B13, H12, MN17 \\
\hline H-ATLAS J142413.9+022303 & G15v2.779 & $193 \pm 9$ & 0.595 & 4.243 & $1.02 \pm 0.04$ & $\mathrm{C} 11, \mathrm{~B} 13, \mathrm{MN} 17$ \\
\hline H-ATLAS J142935.3-002836 & G15v2.19 & $200 \pm 8$ & 0.218 & 1.027 & $0.71 \pm 0.04$ & $\mathrm{C} 14, \mathrm{M} 14, \mathrm{MN} 17$ \\
\hline HeLMS J232439.5-043935 & HeLMS7 & $172 \pm 9$ & - & 2.473 & $0.65 \pm 0.05$ & N16 \\
\hline HeLMS J233255.4-031134 & HeLMS2 & $263 \pm 8$ & 0.426 & 2.689 & $0.93 \pm 0.05$ & N16 \\
\hline HeLMS J233255.6-053426 & HeLMS15 & $147 \pm 9$ & 0.976 & 2.402 & $0.98 \pm 0.05$ & N16 \\
\hline HeLMS J234051.5-041938 & HeLMS5 & $205 \pm 8$ & - & 3.503 & $0.54 \pm 0.05$ & N16 \\
\hline HeLMS J235331.9+031718 & HeLMS40 & $111 \pm 7$ & 0.821 & - & $0.26 \pm 0.05$ & N16 \\
\hline
\end{tabular}

Note. Column $\theta_{\mathrm{E}}$ corresponds to the Einstein radius, which is half the image separation. The references, from which the lens and source redshift were obtained, are as follows: C11 - Cox et al. (2011); B13 - Bussmann et al. (2013); C14 - Calanog et al. (2014); N16 Nayyeri et al. (2016); MN17 - Negrello et al. (2017); M17 - Marchetti et al. (in preparation).

flat $\Lambda$ cold dark matter $(\Lambda \mathrm{CDM})$ model with the best-fitting parameters derived from the results from the Planck Observatory (Planck Collaboration XVI 2014), which are $\Omega_{\mathrm{m}}=0.307$ and $h=0.693$.

\section{THE PILOT SAMPLE AND THE ALMA OBSERVATIONS}

ALMA has much better angular resolution and surface brightness sensitivity than the SMA, making it a much better instrument for mapping a strongly lensed submillimetre source. In our previous SMA study of the lensing statistics of strongly lensed Herschel sources (B13), the limited angular resolution of the SMA meant that it was often not clear whether the structure seen on the maps was actually due to lensing. There is also the possibility that large arcs were missed by their falling below the surface brightness limit of the SMA. Since the new ALMA observations would be so much better than the SMA observations, we defined a new sample of sources for our ALMA programme.

Negrello et al. (2010) showed that it is possible to select a sample of lensed sources from a Herschel survey with close to 100 per cent efficiency. Of the Herschel sources with 500- $\mu$ m flux densities $>100 \mathrm{mJy}$, roughly one-half are strongly lensed and half are a mixture of nearby galaxies and radio-loud AGN. Negrello et al. (2010) showed that it is actually very easy removing these contaminants, since nearby galaxies are easy to identify by inspecting optical surveys, such as the Sloan Digital Sky Survey, and radio-loud AGN are easy to spot because they are found in radio surveys. After rejecting contaminants in this way, Negrello et al. (2010) achieved a 100 per cent success rate for their initial sample.

A number of Herschel teams have used this method to select samples of sources that are probably lensed and then used molecular line spectroscopy to measure redshifts for the sources. A slight variant on the basic method followed by most of these teams is to use the ratios of fluxes in the Herschel bands to select sources that are likely to have redshifts in the wavelength range covered by their spectrometer (e.g. Harris et al. 2012; Lupu et al. 2012), which will create a slight bias towards certain redshift ranges.
An accurate lensing model for a source requires it to have an accurate redshift. Therefore, as the initial sample for our ALMA programme, we selected 42 sources from the H-ATLAS and HeLMS surveys with the highest $500-\mu \mathrm{m}$ flux densities and with spectroscopic redshifts $>1$. We checked that none of our candidates are a radio-loud AGN. In almost all cases, the 500- $\mu \mathrm{m}$ flux densities of the sources are $>100 \mathrm{mJy}$, the flux limit used by Negrello et al. (2010). The lower redshift limit, of course, removes any nearby galaxies, and so we expect virtually all of the sources to be strongly lensed. For the reasons described above, the requirement that the sources have spectroscopic redshifts has probably introduced a slight bias towards certain redshift ranges, but the conditional probability statistics we use in this paper (see Section 4) ensures that our results will not be affected by this bias. Of the 42 sources, only 16 were finally observed by ALMA before the end of Cycle 2, but this should not introduce any bias because we did not rank the sources in priority. Table 1 lists the sample of 16 sources.

We observed each source for approximately 2-4 min with ALMA at $873 \mu \mathrm{m}$ with a maximum baseline of $1 \mathrm{~km}$, which gives an angular resolution of 0.12 arcsec. The final image products were produced by the standard ALMA pipeline. The lensed sources are shown in Fig. 1, all except one. The source H-ATLAS J083344.9+000109 is barely detected in the ALMA image and is the faintest 500- $\mu \mathrm{m}$ source in the sample. There are no obvious signs of lensing features, either on the ALMA image or on the optical image from the Sloan Digital Sky Survey. This source is coincident with a quasi-stellar object (QSO). In addition, the source H-ATLAS J141351.9-000026 does not seem to have any lensing structure. However, as seen from fig. 3 in Negrello et al. (2017) the ALMA image captures a small part of large faint arc.

For the remaining sources in the sample, there is clear evidence of strong lensing features in the ALMA images. Modelling of the submillimetre emission, by constructing detailed lensing models, will be presented in two upcoming papers (Dye et al. 2017; Negrello et al., in preparation). The Einstein radii were measured directly from the images and subsequently compared with the respective values that arise from preliminary lensing models of these systems, whereupon an agreement was confirmed. In cases where only an arc 


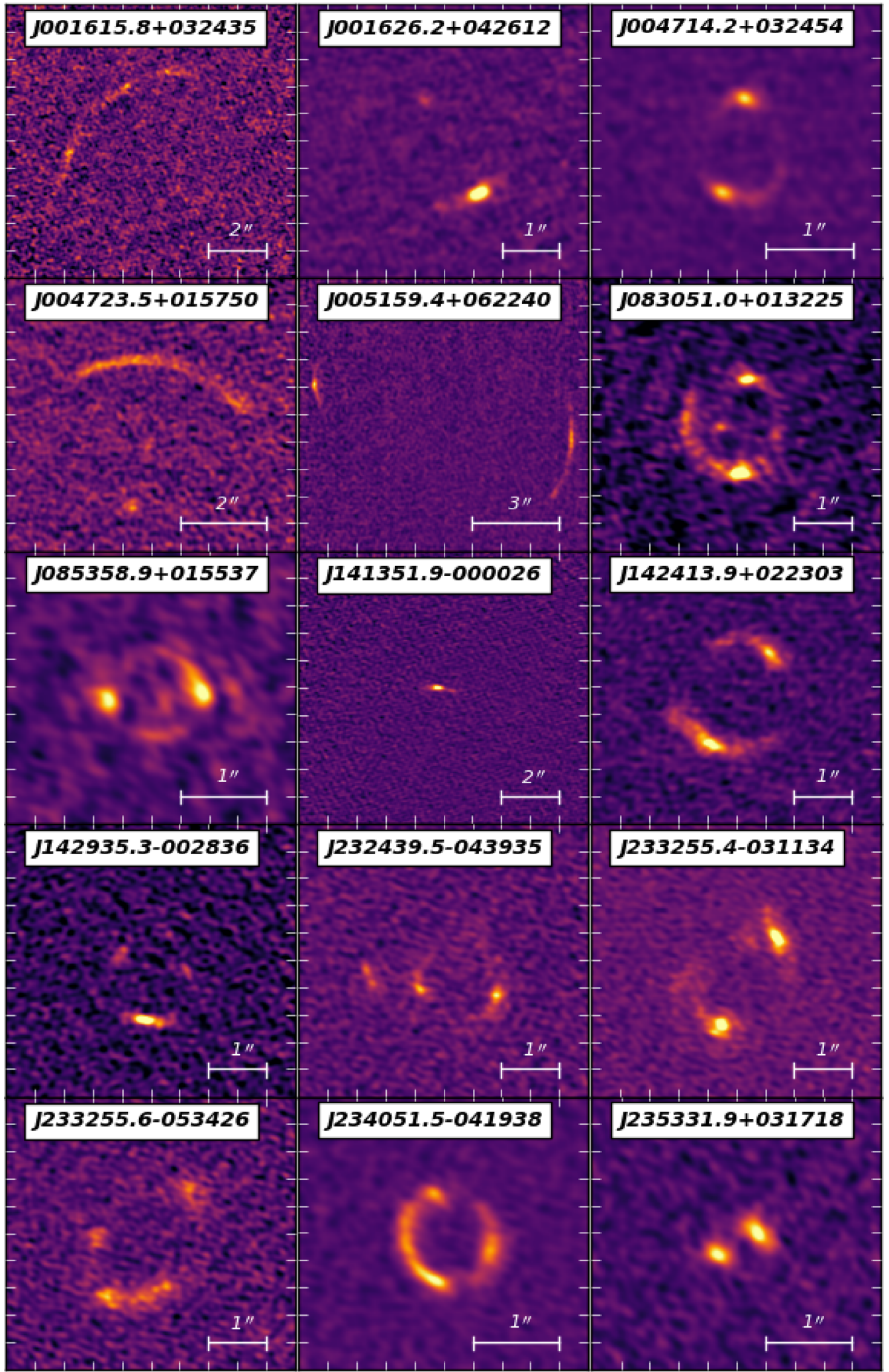

Figure 1. The 873- $\mu \mathrm{m}$ continuum emission images of the 15 sources we observed with ALMA. The source H-ATLAS J083344.9+000109, which was part of the observing run, has been neglected because it does not reveal any lensing features. The flux axes are not shown on the same scale for all the lens systems, as the large arcs would appear very faint. North is up and east is left. 
Table 2. The SMA sample.

\begin{tabular}{lccccc}
\hline IAU name & Name & $z_{\mathrm{l}}$ & $z_{\mathrm{s}}$ & $\theta_{\mathrm{E}}(\operatorname{arcsec})$ & Ref. \\
\hline H-ATLAS J083051.0+013225 & G09v1.97 & 0.6260 & 3.6340 & $0.39 \pm 0.02$ & $\mathrm{~B} 13$ \\
H-ATLAS J085358.9+015537 & G09v1.40 & - & 2.0894 & $0.55 \pm 0.04$ & $\mathrm{~B} 13, \mathrm{M} 17$ \\
H-ATLAS J090302.9-014127 & SDP17 & 0.9435 & 2.3049 & $0.33 \pm 0.02$ & N10, B13 \\
H-ATLAS J090311.6+003906 & SDP1 & 0.2999 & 3.0420 & $1.52 \pm 0.03$ & N10, B13 \\
H-ATLAS J090740.0-004200 & SDP9 & 0.6129 & 1.5770 & $0.59 \pm 0.04$ & N10, B13 \\
H-ATLAS J091043.1-000321 & SDP11 & 0.7930 & 1.7860 & $0.95 \pm 0.02$ & N10, B13 \\
H-ATLAS J091305.0-005343 & SDP130 & 0.2201 & 2.6260 & $0.43 \pm 0.07$ & N10, B13 \\
H-ATLAS J114637.9-001132 & G12v2.30 & 1.2247 & 3.2590 & $0.65 \pm 0.02$ & B13, O13 \\
H-ATLAS J125135.4+261457 & NCv1.268 & - & 3.6750 & $1.02 \pm 0.03$ & B13 \\
H-ATLAS J125632.7+233625 & NCv1.143 & 0.2551 & 3.5650 & $0.68 \pm 0.01$ & B13 \\
H-ATLAS J132427.0+284449 & NBv1.43 & 0.9970 & 1.6760 & - & G05, G13 \\
H-ATLAS J132630.1+334410 & NAv1.195 & 0.7856 & 2.9510 & $1.80 \pm 0.02$ & B13 \\
H-ATLAS J133649.9+291801 & NAv1.144 & - & 2.2024 & $0.40 \pm 0.03$ & B13, O13 \\
H-ATLAS J133542.9+300401 & - & 0.980 & 2.6850 & - & S14, R17 \\
H-ATLAS J133846.5+255054 & - & 0.420 & 2.4900 & - & N17 \\
H-ATLAS J134429.4+303036 & NAv1.56 & 0.6721 & 2.3010 & $0.92 \pm 0.02$ & H12, B13 \\
H-ATLAS J142413.9+022303 & G15v2.779 & 0.5950 & 4.243 & $0.57 \pm 0.01$ & B13 \\
HERMES J021830.5-053124 & HXMM02 & 1.350 & 3.3950 & $0.44 \pm 0.02$ & B13, W13 \\
HERMES J105712.2+565457 & HLock03 & - & 2.7710 & - & W13 \\
HERMES J105750.9+573026 & HLock01 & 0.600 & 2.9560 & $3.86 \pm 0.01$ & B13, W13 \\
HERMES J110016.3+571736 & HLock12 & 0.630 & 1.6510 & $1.14 \pm 0.04$ & C14 \\
HERMES J142825.5+345547 & HBootes02 & 0.414 & 2.8040 & $0.77 \pm 0.03$ & B13, W13 \\
\hline
\end{tabular}

Note. Column $\theta_{\mathrm{E}}$ corresponds to the Einstein radius, which is half the image separation. The references, from which the lens and source redshift were obtained and the estimates for the Einstein radii, are as follows: G05 - Gladders \& Yee (2005); N10 - Negrello et al. (2010); H12 - Harris et al. (2012); B13 - Bussmann et al. (2013); G13 - George et al. (2013); O13 - Omont et al. (2013); W13 - Wardlow et al. (2013); C14 - Calanog et al. (2014); D14 - Dye et al. (2014); M14 Messias et al. (2014); S14 - Stanford et al. (2014); N16 - Nayyeri et al. (2016); M17 - Marchetti et al. (in preparation); R17 - Riechers et al. (in preparation).

is visible (e.g. HeLMS J001615.8+032435) a rough estimate of the Einstein radius was performed by fitting a circle to the peaks of the emission $(>4 \sigma)$. A uniform weighting was applied to these pixels, alleviating any dependence on their fluxes and taking into account only on their positions.

For three sources (H-ATLAS J083051.0+013225, H-ATLAS J085358.9+015537, and H-ATLAS J142413.9+022303) there are also measurements of the Einstein radius from SMA observations (see Table 2). For these sources, the pairs of measurements, with the SMA measurement first are: $0.39 \pm 0.02$ and $0.85 \pm 0.04$ arcsec; $0.55 \pm 0.04$ and $0.55 \pm 0.04$ arcsec; $0.57 \pm 0.01$ and $1.02 \pm 0.04 \mathrm{arcsec}$. This disagreement in the inferred values of the Einstein radii can be attributed to the complex structure of the submillimetre emission that cannot be fully resolved with the SMA observations, as well as the complexity of the foreground mass distribution (B13).

\section{METHODOLOGY}

In this section we describe the methodology for predicting the distribution of image separations. In Section 3.1 we discuss the different density profiles that were considered in this work. In Section 3.2 we present the halo mass function model. In Section 3.3 we describe the standard approach for computing lensing properties assuming spherical symmetry, and finally in Section 3.4 we lay down the formalism for computing strong lensing statistics.

\subsection{The halo density profiles}

In the dark matter halo paradigm, galaxies are forming in an evolving population of dark matter haloes. High-resolution pure dark matter $N$-body simulations have been used extensively to study this dark component of the Universe. These studies suggest that the spatial mass density distribution of dark matter, inside the haloes identified in simulations, is well fitted by a single profile across a wide range of halo masses, the Navarro-Frenk-White (NFW) profile (Navarro, Frenk \& White 1996, 1997). The NFW density profile is given by

$$
\rho(r)=\frac{\rho_{\mathrm{s}}}{\left(r / r_{\mathrm{s}}\right)\left(1+r / r_{\mathrm{s}}\right)^{2}},
$$

where $r_{\mathrm{s}}=r_{\mathrm{vir}} / c$ is the scale radius with $c$ being the concentration parameter that is approximated by the formula

$c(M, z)=5\left(\frac{M_{\mathrm{h}}}{10^{13} \mathrm{M}_{\odot}}\right)^{-0.074}\left(\frac{1+z}{1.7}\right)^{-1}$,

and is derived from numerical simulations of Prada et al. (2012).

However, the objects that we observe in the real universe are composed of both dark and baryonic matter. The difficulty is in producing density profiles for haloes that also include baryons, because the physics of how baryons accrete into the centre of the halo and the astrophysical processes that take place in these central regions are complex and poorly understood. Two different analytic approaches are considered in this study, in an attempt to describe the total mass density distribution in early-type galaxies.

The simplest approach that is frequently used in the literature is the Singular Isothermal Sphere (SIS) model. The SIS density profile is given by

$\rho(r)=\frac{\sigma_{v}^{2}}{2 \pi G r^{2}}$,

where $G$ is the gravitational constant and $\sigma_{v}$ is the velocity dispersion of the halo. The later can be determined from the circular 
velocity of the halo, $V^{2}=G M_{\mathrm{h}} / r_{\mathrm{vir}}$, following the commonly used assumption that $\sigma_{v} \approx V / \sqrt{2}$. There are strong observational evidence that this power-law model provides a good description of the total mass distribution in field early-type galaxies. Joint gravitational lensing and stellar dynamical analysis of a sample of strong lenses from the SLACS Survey does indeed confirm that the average logarithmic slope for the total mass density is $\langle\gamma\rangle \simeq 2.0$ with some intrinsic scatter (Koopmans et al. 2006, 2009). Similar analysis was performed for the first five strong gravitational lens systems discovered in H-ATLAS (Dye et al. 2014), where the results found were in agreement with previous studies.

Recently, Lapi et al. (2012) adopted a rather theoretical approach by considering the contribution from baryons and dark matter, separately. They used an NFW profile to represent the mass density distribution for the dark matter component and a Sérsic profile for the stellar component. The three-dimensional functional form of the Sérsic profile (Prugniel \& Simien 1997) is given by

$\rho(r)=\frac{M_{\star}}{4 \pi R_{\mathrm{e}}^{3}} \frac{b_{n}^{2 n}}{n \Gamma(2 n)}\left(\frac{r}{R_{\mathrm{e}}}\right)^{-\alpha_{n}} \exp \left[-b_{n}\left(\frac{r}{R_{\mathrm{e}}}\right)^{1 / n}\right]$,

where $n$ is the Sersic index, $R_{\mathrm{e}}$ is the effective radius, $b_{n}=2 n-1 / 3+0.009876 / n, a_{n}=1-1.188 / 2 n+0.22 / 4 n^{2}$, and $M_{\star}$ is the stellar mass. The stellar mass can be determined by assuming a fixed ratio between the halo and stellar mass $M_{\mathrm{h}} / M_{\star}$.

Lapi et al. (2012) showed that for galaxy-scale lenses this model, hereafter referred to as the SISSA model, yields very similar results to the SIS model under the assumption of reasonable parameters. However, this model has two additional free parameters that are affected by a large scatter. The first parameter is the ratio of halo to stellar mass, which for early-type galaxies is expected to lie in the range of 10-70. The second parameter is the concentration parameter, $c$, which is expected to have a 20 per cent scatter. In our analysis we will omit the scatter in the $c-M$ relation and adopt a constant ratio of halo to stellar mass of 30 . However, we show in Appendix how these parameters can affect our results.

An additional parameter that is introduced in the abovementioned models is the virialization redshift $z_{1, v}$. This parameter is used to determine the virial radius of the halo $r_{\mathrm{vir}}$,

$r_{\mathrm{vir}}=\left(\frac{3 M_{\mathrm{h}}}{4 \pi \Delta_{\mathrm{c}} \rho_{\text {crit }}}\right)^{1 / 3}$,

where $\rho_{\text {crit }}(z)=\rho_{\text {crit }, 0} E^{2}(z)$ is the critical density of the universe at redshift $z$, with $\rho_{\text {crit }, 0}$ being its value at redshift zero and $E(z)$ is the scaled Hubble parameter,

$E^{2}(z)=\frac{H^{2}(z)}{H_{0}^{2}}=\Omega_{\mathrm{m}, 0}(1+z)^{3}+\Omega_{\Lambda, 0}(1+z)^{3(1+w)}$.

Assuming a flat cosmology $\left(\Omega_{\mathrm{m}}+\Omega_{\Lambda}=1\right)$ we can use an approximate expression for $\Delta_{c}$, which was derived from a fit to simulations of Bryan \& Norman (1998),

$\Delta_{\mathrm{c}}=18 \pi^{2}+82 x-39 x^{2}$,

where $x=\Omega_{\mathrm{m}}(z)-1$ and the redshift evolution of the cosmological parameter of matter is

$\Omega_{\mathrm{m}}(z)=\frac{\rho_{\mathrm{m}}}{\rho_{\text {crit }}}=\Omega_{\mathrm{m}, 0}(1+z)^{3} / E^{2}(z)$.

Lapi et al. (2012) suggested that the frequently made approximation that the observed redshift of a galaxy is equal to the virialization redshift $z_{1} \approx z_{1, \mathrm{v}}$ leads to an overestimation of the halo size. Alternatively they propose a virialization redshift in the range $z_{1, \mathrm{v}} \sim 1.5-3.5$, which is much more in line with the ages of the stellar populations found in early-type galaxies.

Besides the analytic models presented above, we also now have results from cosmological hydrodynamic simulations that provide the means to examine how baryonic effects modify the structure of dark matter haloes in a more rigorous way. In recent studies, Schaller et al. $(2015 \mathrm{a}, \mathrm{b})$ investigated the internal structure of haloes produced in the Evolution and Assembly of GaLaxies and their Environments (EAGLE) simulations, which include both baryons and dark matter (Schaye et al. 2015). Some of the baryonic effects that are included in these simulation runs are feedback processed from massive stars and AGN, radiative cooling, and contraction of the dark matter in the central halo regions due to the presence of baryons. The authors demonstrated that the following formula

$\frac{\rho(r)}{\rho_{\text {crit }}}=\frac{\delta_{\mathrm{s}}}{\left(r / r_{\mathrm{s}}\right)\left(1+r / r_{\mathrm{s}}\right)^{2}}+\frac{\delta_{\mathrm{i}}}{\left(r / r_{\mathrm{i}}\right)\left(1+\left(r / r_{\mathrm{i}}\right)^{2}\right)}$

provides a good fit to the data. From the above functional form we clearly see that the first term is the NFW profile that provides a fairly good description of the outer part of the halo. The second term is an NFW-like profile with a steeper slope to account for the concentration of baryons in the central region of the halo. The parameters of this model as a function of mass, namely $\delta_{\mathrm{s}}, r_{\mathrm{s}}, \delta_{\mathrm{i}}$, and $r_{i}$, are determined by fitting third-order polynomials to the values found in table 2 of Schaller et al. (2015a). The halo mass range probed in this study ranges from $M_{\mathrm{h}}=10^{10}$ to $10^{14} \mathrm{M}_{\odot}$.

\subsection{Halo mass function}

The halo mass function describes the comoving number density of dark matter haloes as a function of redshift and per comoving mass interval. In our earlier paper (Eales 2015), we used analytic functions, obtained by fitting to the results of numerical simulations of the evolution of dark matter, of Sheth \& Tormen (1999) and Tinker et al. (2008; hereafter T08). We found very little difference between the results predicted from the two halo mass functions. Both these analytic functions were based on numerical simulations containing only dark matter. In this paper, we use the analytic function for the halo mass function that was derived by Bocquet et al. (2016) by fitting to the results of a numerical simulation that contains both baryons and dark matter, using the same formalism as T08. The comoving number density of haloes of mass $M$ is given by

$\frac{\mathrm{d} n}{\mathrm{~d} M}=f(\sigma) \frac{\bar{\rho}_{\mathrm{m}}}{M} \frac{\mathrm{d} \ln \sigma^{-1}}{\mathrm{~d} M}$,

where $\bar{\rho}_{\mathrm{m}}$ is the mean number density at the current epoch and $\sigma$ is the square root of the variance of the mass density field,

$\sigma^{2}=\left\langle\left(\frac{\delta M}{M}\right)^{2}\right\rangle=\frac{1}{2 \pi^{2}} \int P^{\operatorname{lin}}(k, z) \hat{W}^{2}(k R) k^{2} \mathrm{~d} k$,

which is smoothed on a scale of comoving radius $R=$ $\left(3 M / 4 \pi \bar{\rho}_{\mathrm{m}, 0}\right)^{1 / 3}$, using the Fourier transform of the real-space tophat filter,

$\hat{W}(k R)=3 \frac{\sin (k R)-(k R) \cos (k R)}{(k R)^{3}}$.

The function $f(\sigma)$ is parametrized as

$f(\sigma)=A\left[\left(\frac{\sigma}{b}\right)^{-\alpha}+1\right] \mathrm{e}^{-c / \sigma^{2}}$,

where the parameters $A, \alpha, b$, and $c$ are all expressed as functions of redshift $A(z)=A_{0}(1+z)^{A_{z}}, \alpha(z)=\alpha_{0}(1+z)^{\alpha_{z}}$, 


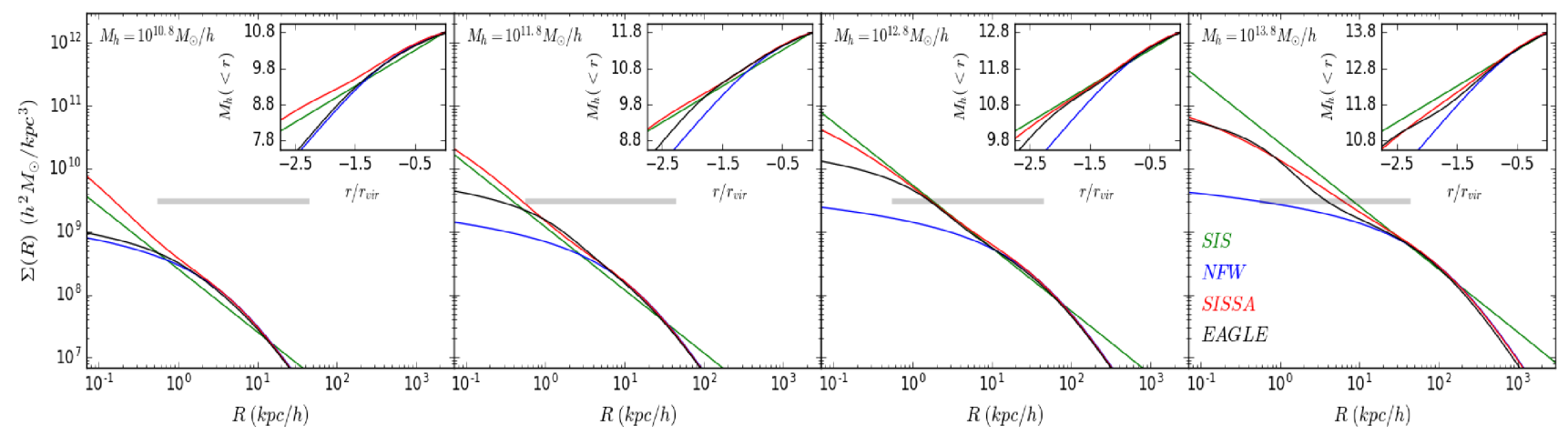

Figure 2. Surface mass density as a function of the radial distance in the lens plane for the different lens models: SIS (green line), NFW (blue line), SISSA (red line), and a halo profile derived from the EAGLE simulation (black line). The grey solid line corresponds to the critical surface density $\Sigma_{\mathrm{c}}$ for $z_{\mathrm{l}}=0.5$ and $z_{\mathrm{s}}=2.0$. The figure insets show the mass enclosed within radius $r$, where the $x$-axis is scaled by the virial radius $r_{\mathrm{vir}}$.

$b(z)=b_{0}(1+z)^{b_{z}}$, and $c(z)=c_{0}(1+z)^{c_{z}}$. The best-fitting values of these parameters are obtained from table 2 of Bocquet et al. (2016) for the Hydro simulation.

\subsection{Lensing properties}

In our analysis we consider the typical lensing configuration that is composed of a point-like source located at redshift $z_{\mathrm{s}}$, an object acting as a lens located at redshift $z_{1}$, and an observer, in order to derive the lensing properties (Schneider et al. 1992). We always assume that the lens is spherically symmetric, since ellipticity does not significantly affect the statistics of image separations (Huterer, Keeton \& Ma 2005).

\subsubsection{Surface density}

The surface density $\Sigma$ can be computed by integrating the 3D density profile of the halo $\rho(r)$ over the parallel coordinate along the line of sight, and expressed as a function of the perpendicular coordinate in the lens plane (thin lens approximation):

$\Sigma(R)=2 \int_{R}^{\infty} \mathrm{d} r \frac{r}{\sqrt{r^{2}-R^{2}}} \rho(r)$.

The condition for strong lensing to occur is that the surface mass density exceeds the critical threshold (critical surface density),

$\Sigma_{\mathrm{c}}=\frac{c^{2}}{4 \pi G} \frac{D_{\mathrm{s}}}{D_{\mathrm{ls}} D_{\mathrm{l}}}$,

which solely depends on the angular diameter distances from the observer to the lens and source plane, corresponding to $D_{1}$ and $D_{\mathrm{s}}$, respectively, as well as the angular distance between lens and source plane $D_{\mathrm{ls}}$. The angular diameter distance is given by

$D_{\mathrm{i}}=\frac{1}{\left(1+z_{\mathrm{i}}\right)} \int_{0}^{z_{\mathrm{i}}} \frac{c \mathrm{~d} z}{H(z)}$.

This expression holds in the case where a flat cosmology is assumed.

Fig. 2 shows the radial dependence of the surface mass density for the various halo density profiles that were considered in this work. The critical surface density, when the source is at redshift $z_{\mathrm{s}}=2.0$ and the lens at $z_{1}=0.5$, is also shown in the figure as the grey solid line. The different panels of the figure correspond to different halo masses (shown in their upper left-hand corner). Note that the maximum resolution of the EAGLE simulation is $\sim 1 \mathrm{kpc}$, below which there is no guarantee their fit is realistic. Each panel has an inset plot showing the mass enclosed within a certain radius.
In low-mass haloes $\left(M_{\mathrm{h}}<10^{11.5} \mathrm{M}_{\odot}\right)$ the predictions from the EAGLE simulation agrees very well with the NFW profile. This range of halo masses corresponds to dwarf galaxies, where the baryon fraction of stellar to halo mass is very low and the dark matter dominates the mass budget. The critical surface density indicated that haloes in this range are very inefficient lenses, not being able to produce multiple images. The SISSA model still predicts that there are baryons in these haloes, but concentrated in lower radial scales beyond the probed range of the EAGLE simulation.

In intermediate-mass haloes $10^{11.5}<M_{\mathrm{h}}<10^{13.5} \mathrm{M}_{\odot}$ the EAGLE density profile gradually departs from the NFW model as baryons start to play an important role. This range of halo masses corresponds to typical early-type galaxies, where the baryon fraction peaks causing baryonic effects to be more prominent. The dense central regions in these objects, which result from the contribution of baryons, makes them very efficient lenses. There is a fairly good agreement between the EAGLE model and both SIS and SISSA models in this range.

In high-mass haloes $M_{\mathrm{h}}>10^{13.5} \mathrm{M}_{\odot}$ the EAGLE model agrees fairly well with the NFW model for radii larger than about $\sim 10 \mathrm{kpc}$, while their central regions are still dominated by the presence of baryons. This range of halo masses corresponds to groups/clusters of galaxies, where the baryon fraction gradually decreases until it reaches the universal mean value $f_{\mathrm{b}}=\Omega_{\mathrm{b}} / \Omega_{\mathrm{m}}$. The SISSA model in this range produces denser central regions as expected, since it is not intended for the description of groups/clusters of galaxies (does not account for the increase in the ratio of halo to stellar mass as the halo grows).

\subsubsection{Image separation}

Assuming that light rays are coming from a distant point-like source and crossing the lens plane at an angular position $\theta$, they will get deflected by an angle $\alpha(\theta)$ that is given by

$\alpha\left(\theta \mid z_{1}, z_{\mathrm{s}}, M_{\mathrm{h}}\right)=\frac{2}{\theta} \int_{0}^{\theta} \theta \mathrm{d} \theta \frac{\Sigma\left(D_{1} \theta \mid z_{1}, M_{\mathrm{h}}\right)}{\Sigma_{c}\left(z_{1}, z_{\mathrm{s}}\right)}$.

This property strongly depends on the mass enclosed within the radius $R \equiv D_{1} \theta$. The true and observed positions of the source in the sky are related through the simple transformation from the lens to the source plane,

$\beta(\theta)=\theta-\frac{\theta}{|\theta|} \alpha(|\theta|)$, 


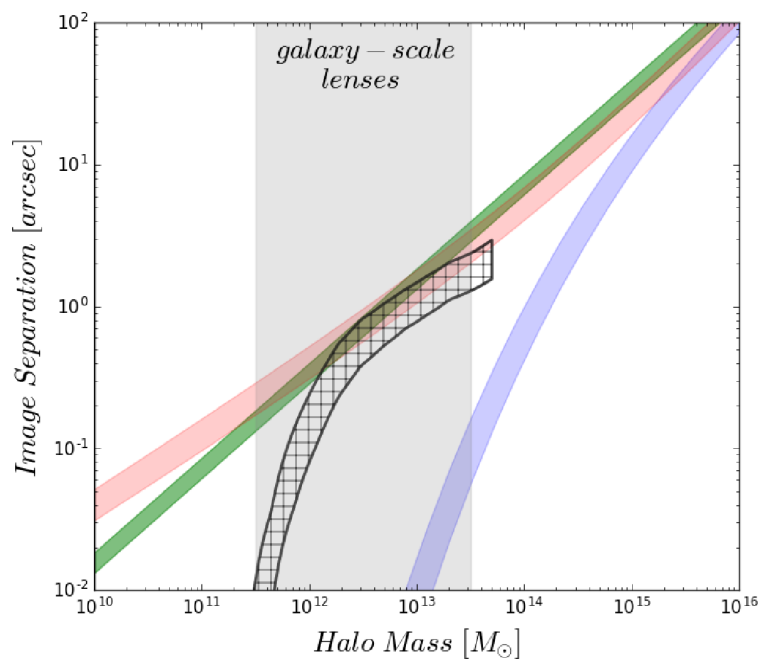

Figure 3. The image separation $\theta$, as a function of the halo mass for the different lens models: SIS (green), NFW (blue), SISSA (red), and EAGLE (black hatched). The width of the stripes correspond to a lens redshift range $z_{1}=0.5-1.0$, while the redshift of the source is kept fixed to $z_{\mathrm{s}}=2.0$. The virialization redshift is assumed to be equal to the redshift of the lens $z_{1, \mathrm{v}}=z_{\mathrm{l}}$ in this case.

referred to as the lens equation. The solutions of the lens equation $\theta_{\mathrm{i}}$, given the position of the source $\beta$ in the source plane, will give the positions of the lensed images in the lens plane. The magnification of individual images can then be computed from

$\mu\left(\theta_{\mathrm{i}} \mid z_{1}, z_{\mathrm{s}}, M_{\mathrm{h}}\right)=\frac{1}{\lambda_{\mathrm{r}} \lambda_{\mathrm{t}}}$,

with

$\lambda_{\mathrm{r}, \mathrm{t}}=1-\kappa\left(\theta_{\mathrm{i}}\right) \pm \gamma\left(\theta_{\mathrm{i}}\right)$,

where the quantities $\kappa(\theta)=\Sigma(\theta) / \Sigma_{\mathrm{c}}$ and $\gamma(\theta)=\alpha(\theta) / \theta-\kappa(\theta)$ are the convergence and shear, respectively, given as a function of the angular position in the lens plane. Therefore, the total magnification of the source, at position $\beta$ in the source plane, is computed by summing up the absolute values of the magnifications of the individual images $\mu_{\mathrm{i}}$ that are formed.

The quantities $\lambda_{r, t}$ in the denominator of equation (19) define the radial and tangential critical curves in the lens plane, where the magnification diverges (when $\lambda_{\mathrm{r}, \mathrm{t}}$ become zero). The Einstein radius for a specific halo density profile corresponds to the radius tangential critical curve, from which we compute the image separation for a set of lens and source parameters as twice its value. Fig. 3 shows how the image separation changes as a function of the halo mass for the different halo models. We can see that EAGLE predicts far smaller image separations for lenses with a mass $10^{11.5}<M_{\mathrm{h}}<10^{12.5} \mathrm{M}_{\odot}$ compared to the SIS and SISSA models, while in the range $10^{12.5}<M_{\mathrm{h}}<10^{13.5} \mathrm{M}_{\odot}$ there is a good agreement.

\subsubsection{Cross-section}

The most important quantity for studies of strong lens statistics is the cross-section. This is defined as the area in the source plane where the source has to lie in order to have a total magnification of $>\mu$. For a spherically symmetric mass distribution the cross-section can be easily computed by

$\sigma\left(\geq \mu, z_{1}, z_{\mathrm{s}}, M_{\mathrm{h}}\right)=\pi \beta^{2}(\mu)$,

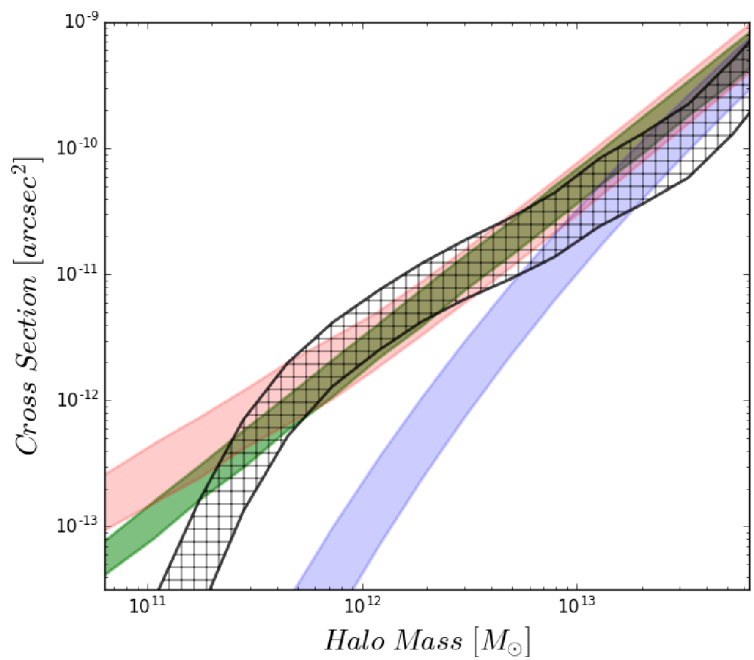

Figure 4. The cross-section $\sigma(\mu>2)$, as a function of the halo mass for the different lens models: SIS (green), NFW (blue), SISSA (red), and EAGLE (black hatched). The width of the stripes corresponds to a lens redshift range $z_{1}=0.5-1.0$, while the redshift of the source is kept fixed to $z_{\mathrm{s}}=2.0$. The virialization redshift is assumed to be equal to the redshift of the lens $z_{1, \mathrm{v}}=z_{1}$ in this case. The range of halo mass corresponds to the grey highlighted area in Fig. 3 of galaxy-scale lenses.

where $\beta(\mu)$ is the radius in the source plane corresponding to a magnification $\mu$.

We calculated the cross-section using a minimum magnification factor of $\mu_{\min }=2$. For the SIS model, this corresponds to the stronglensing regime in which multiple images are produced. We used the same minimum magnification factor for the other density profiles, even though this is not the magnification at which multiple images start to be seen. This was partly for consistency but also because we did not originally select our sample of lensed sources because they had multiple images but because their flux densities were amplified enough to be detected in a sample of bright $500-\mu \mathrm{m}$ sources.

Fig. 4 shows the behaviour of the cross-section as a function of the halo mass for the different halo models, only for the range of galaxyscale lenses. As illustrated in the figure, for the range of masses relevant to galaxy-scale lenses $10^{11.5}<M_{\mathrm{h}}<10^{13.0} \mathrm{M}_{\odot}$, there is an agreement between the SIS, SISSA, and EAGLE models. As the halo mass grows above $10^{13.0} \mathrm{M}_{\odot}$ the EAGLE's cross-section behaviour starts to divert from these and slightly becomes similar to that of the NFW model.

\subsubsection{Magnification bias}

'Magnification bias' leads to lensed systems being over-represented in a flux-limited or magnitude-limited sample because there are more low-luminosity sources in the universe, which lensing can boost over the flux limit, than high-luminosity sources (e.g. Eales 2015; Mason et al. 2015). Our study is immune to this effect because our statistical methodology (Section 3.4) is based on the assumption that we have found (it does not matter in what way) a sample of lensed sources, and we then consider the conditional probability of the Einstein radius given a particular source redshift. However, because shallower density distributions produce larger magnifications, magnification bias could potentially distort the distributions of Einstein radii that we measure. We have modelled this bias in the following way. 


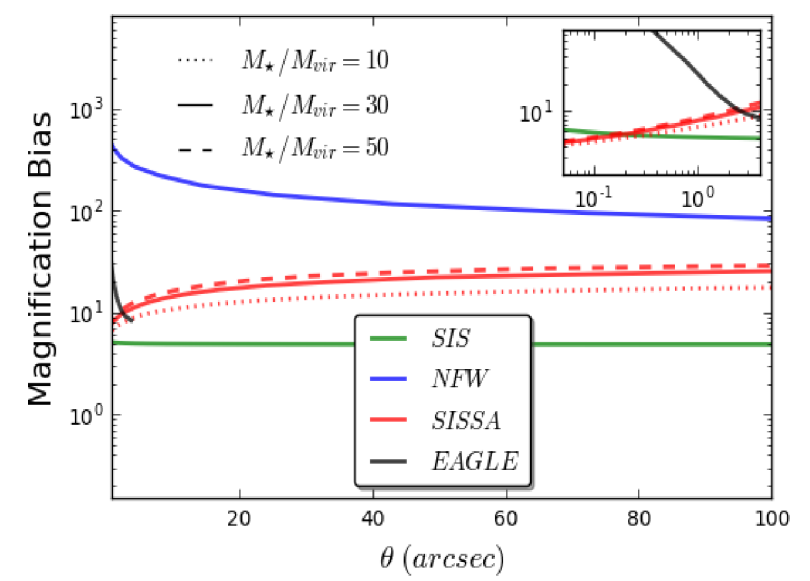

Figure 5. The magnification bias as a function of the image separation, computed for a luminosity function $\Phi(L) \propto L^{-2.1}$. The calculation is performed for different lens model: SIS (green), NFW (blue), SISSA (red), and EAGLE (black). The various red lines correspond to the SISSA model adopting different choices for the ration of stellar to halo mass. The inset plot shows a zoom in to the smaller angular scales.

The magnification bias causes sources that are fainter than the limiting magnitude of the survey to be detected in the sample. We define this bias factor as

$B\left(L \mid z_{\mathrm{s}}\right)=\frac{2}{\beta_{\mathrm{r}}^{2}} \int_{0}^{\beta_{\mathrm{r}}} \beta \frac{\Phi\left(L / \mu(\beta) \mid z_{\mathrm{s}}\right)}{\Phi\left(L \mid z_{\mathrm{s}}\right)} \frac{\mathrm{d} \beta}{\mu(\beta)}$,

where $\Phi\left(L \mid z_{\mathrm{s}}\right)$ is the luminosity function. We calculate how the bias factor depends on image separation for the different density distributions. We assume that the luminosity function follows a power law with the form $\Phi\left(L \mid z_{\mathrm{s}}\right) \propto L^{-2.1}$, which is a good approximation to the form of the submillimetre luminosity function at high luminosities (Gruppioni et al. 2013), and we assume this is the same for all source redshifts.

Fig. 5 shows the computed magnification bias as a function of the image separation for the different lens models. Although, in principle, we could use our models to correct for this effect, we have decided not to do this because the luminosity function for submillimetre sources is still very poorly constrained, and so the model is very uncertain. Fig. 5 shows that there will be no effect for the SIS model, because the magnification bias is independent of angular image separation, but the effect for the other density profiles may be significant.

\subsection{Formalism of strong lens statistics}

We adopt the standard formalism for computing lensing statistics (Turner, Ostriker \& Gott 1984), where we consider a population of dark matter haloes that act as deflectors located at redshift $z_{1}$ and can be characterized by their mass $M_{\mathrm{h}}$. The differential probability that a source at redshift $z_{\mathrm{s}}$ is strongly lensed with total magnification $\geq \mu$ by that population of deflectors is given by

$$
\frac{\mathrm{d} P}{\mathrm{~d} z_{1} \mathrm{~d} M_{\mathrm{h}}}=\frac{\mathrm{d}^{2} N}{\mathrm{~d} M_{\mathrm{h}} \mathrm{d} V} \frac{\mathrm{d}^{2} V}{\mathrm{~d} z_{1} \mathrm{~d} \Omega} \sigma\left(\geq \mu, z_{1}, z_{\mathrm{s}}, M_{\mathrm{h}}\right),
$$

where

$$
\frac{\mathrm{d}^{2} V}{\mathrm{~d} z_{1} \mathrm{~d} \Omega}=\frac{c}{H_{0}} \frac{\left(1+z_{1}\right)^{2} D_{\mathrm{A}}^{2}\left(z_{1}\right)}{E\left(z_{1}\right)}
$$

is the comoving volume element per unit of $z_{1}$ interval and solid angle, while $\mathrm{d}^{2} N / \mathrm{d} M_{\mathrm{h}} \mathrm{d} V$ is the number density of deflectors per units of $M_{\mathrm{h}}$ interval at different redshifts.

The total lensing probability $P\left(z_{\mathrm{s}}, \geq \mu\right)$ can be computed by integrating equation (23) over the lens redshift and halo mass ranges. To calculate the probability distribution of image separations we insert a selection function in the integral in order to select only the combination of parameters that produce image separations in the interval $\theta \pm \mathrm{d} \theta$. The probability distribution as a function of the image separation then becomes

$$
P\left(\theta \mid z_{\mathrm{s}}, \geq \mu\right)=\int_{0}^{z_{\mathrm{s}}} \mathrm{d} z_{1} \int_{0}^{\infty} \mathrm{d} M_{\mathrm{h}} \frac{\mathrm{d} P}{\mathrm{~d} z_{1} \mathrm{~d} M_{\mathrm{h}}} \delta\left[\theta-\tilde{\theta}\left(z_{1}, z_{\mathrm{s}}, M_{\mathrm{h}}\right)\right],
$$

where $\tilde{\theta}\left(z_{1}, z_{\mathrm{s}}, M_{\mathrm{h}}\right)$ is calculated for each model as twice the Einstein radius (tangential critical curve) and the Dirac $\delta$ function is unity if the combination of parameters corresponds to image separation $\tilde{\theta}$ in the interval $(\theta-\mathrm{d} \theta, \theta+\mathrm{d} \theta)$.

The amplitude of the image separation distribution in equation (25) increases with increasing source redshift independently of the angular scale, since we sample a larger volume of the universe. The normalized image separation distribution on the other hand,

$$
p\left(\theta \mid z_{\mathrm{s}}, \geq \mu\right)=\frac{P\left(\theta \mid z_{\mathrm{s}}, \geq \mu\right)}{\int_{0}^{\infty} \mathrm{d} \theta P\left(\theta \mid z_{\mathrm{s}}, \geq \mu\right)},
$$

is quite insensitive to the source population and the cosmological parameters (Oguri 2002). Comparing the predicted normalized distribution with the observed one, we therefore probe the combination of the halo mass function and density profiles of haloes that affect the shape of the distribution.

In our analysis we assume a two-transition mass model, following the methodology adopted in previous studies (Porciani \& Madau 2000; Kochanek \& White 2001; Oguri 2002; Kuhlen, Keeton \& Madau 2004). This approach was introduced in order to account for baryons, which probably affect the shape of halo's density profile by means of adiabatic contraction (Blumenthal et al. 1986) and cooling (White \& Rees 1978) when the baryon fraction is relatively high. In our model, haloes below the mass $M_{\text {min }}$ (corresponding to dwarf galaxies) and above $M_{\max }$ (corresponding to clusters of galaxies) are described by the NFW profile to account for the expected low baryon fraction. In the intermediate-mass range (corresponding to early-type galaxies) haloes are described by either the SIS or SISSA model, where the baryon fraction is expected to reach the peak.

Another quantity that was introduced in the analytic description of the SIS and SISSA models in Lapi et al. (2012) is the virialization redshift of the lens $z_{\mathrm{l}, \mathrm{v}}$. According to their study, the frequently made approximation $z_{1, \mathrm{v}} \approx z_{1}$ leads to an underestimate of the lensing probability. This is because a lower value of the virialization redshift leads to an overestimation of the halo size and therefore to an underestimation of the halo's density. As a result, a higher upper transition mass would be necessary in order to match the observed distribution of image separations. We examine the effect of the virialization redshift on the transition masses of our model by considering both a $z_{1, \mathrm{v}}=z_{1}$ and $z_{1, \mathrm{v}}=2.5$ (see Lapi et al. 2012 for details) when computing the theoretical distribution of image separations.

\section{RESULTS}

In this section we follow the methodology described in Section 3, to derive the theoretical distributions of image separations. We then compare our model predictions with the normalized histogram of the 
observed image separations for two samples of Herschel-selected lensed sources. We emphasize that the use of the conditional probability distribution means that our analysis is independent of the properties of the source population. We carry out the analysis separately for the sample of sources observed with ALMA and SMA.

\subsection{Comparison with observations}

We derive the values of our two transition-mass models, described in Section 3.4, by performing a standard $\chi^{2}$ minimization method:

$\chi^{2}=\sum_{i} \frac{\left(P\left(\theta_{\mathrm{i}} \mid \geq \mu\right)-P^{\prime}\left(\theta_{\mathrm{i}} \mid \geq \mu\right)\right)^{2}}{\sigma^{2}\left(\theta_{\mathrm{i}}\right)}$,

where $P(\theta)$ and $P^{\prime}(\theta)$ are the observed and theoretical normalized image separation distributions, respectively. The quantity $\sigma(\theta)$ is the standard deviation of each bin of the observed histogram of image separations, which is derived from Poisson statistics.

Fig. 6 shows a comparisons of the observed and predicted distributions of image separations. The black solid line shows the predicted distribution using the analytic mass density distribution obtained from the EAGLE simulation (equation 9). This agrees fairly well with the observations, and does not require the imposition of transition masses. The other lines show the predictions of our analytic models with two transition masses. The graphs show our predictions adopting a virialization redshift $z_{1, \mathrm{v}}=z_{1}$ and $z_{1, \mathrm{v}}=2.5$ as straight and dashed lines, respectively.

The grey histograms in each graph correspond to the observed distributions for the sample of sources observed with ALMA on the left-hand side, and with the sample of sources observed with SMA that was used in our previous study (Eales 2015), on the right-hand side. The best-fitting values of the two transition masses are shown in Table 3 for the two different choice of virialization redshift along with the different choices of halo density profiles and observed sample. In order to account for the uncertainty on the measured image separations, we perform 100 simulations for each measurement by resampling each value at random from a Gaussian distribution with a standard deviation equal the value's error. For each realization of the observed distribution we perform the above fitting procedure and we end up with a distribution for the upper transition mass from which we derive its errors.

In our analysis we decided to exclude the object J141351.9-000026, which as discussed in Section 2 has a very large Einstein radius as a result of lensing by a galaxy cluster. If we were to include this object in the analysis there would not be any significant difference in the constrained value of the maximum transition masses. This is because the constrain is more sensitive to the contribution from the galaxy scale lenses. Increasing the maximum transition mass will shift the kink of the distribution to larger scales and the lack of objects in that range constrains its value. Including an object with significantly larger Einstein radius than where the kink is observed will not significantly contribute to the fitting method. Furthermore, no proper modelling has been performed for this object to extract the value of its Einstein radius.

Predictions adopting either of the analytic profiles, SIS and SISSA, as well as the density profile derived from the EAGLE simulation, seem to be in good agreement with observations. Furthermore, comparing the fitted values of the upper transition mass that were obtained for the different samples of lenses, we find a slight difference that is not significant (i.e. $<1 \sigma$ ). As mentioned in Section 2, the observed distribution of image separations, for the SMA sample, is biased towards lower angular separations, which leads to an underestimate of the upper transition mass. Concerning the lower transition mass, we are still not in a position to set good constrains because our fitting method cannot distinguish models with $M_{\min } \lesssim 10^{12.5} \mathrm{M}_{\odot}$. Finally, the virialization redshift strongly affects the resulting transition masses, pushing them to lower values. However, there is still no evidence to support such a low-transition mass between galaxies and clusters.

\section{DISCUSSION AND CONCLUSIONS}

Wide-area extragalactic surveys conducted at submillimetre wavelengths have allowed us to discover a new population of strongly lensed galaxies (Negrello et al. 2010, 2017; Nayyeri et al. 2016). Their potential to produce very large samples of strong lenses (González-Nuevo et al. 2012) and the simplicity of the selection function (Blain 1996; Perrotta et al. 2002, 2003; Negrello et al. 2007) will greatly benefit the study of strong lens statistics, a subject that has previously been studied by optical (Bolton et al. 2006; More et al. 2012) and radio surveys (Browne et al. 2003; Oguri et al. 2006). Extragalactic surveys undertaken with Herschel Space Observatory have demonstrated the potential of this method by producing large samples of candidate strong lenses (Wardlow et al. 2013; Nayyeri et al. 2016; Negrello et al. 2017). We carried out follow-up observations with ALMA of 16 candidate strongly lensed Herschel sources, selected from the H-ATLAS and HeLMS surveys, expecting that based on their bright 500- $\mu \mathrm{m}$ flux densities that they should be lensed. Out of these sources, 15 show clear evidence of lensing features.

In this study we predict the distribution of image separations of strongly lensed systems produced by a population of dark matter haloes parametrized by the halo mass function derived from hydrodynamical cosmological simulations (Bocquet et al. 2016). The largest uncertainty that enters the calculation of the theoretical image separation distribution is the total mass distribution of these haloes, which is the primary focus of this study. For the first time we used a halo density profile that was derived from the EAGLE simulation (Schaller et al. 2015a,b), which is calibrated so that it provides a good fit across a wide range of halo masses. We showed that the combination of mass density distributions and the halo mass function predicted by cosmological numerical simulations can reproduce the observed distribution of image separation of strong lenses found in submillimetre surveys.

We also consider a different approach adopting analytical recipes for the description of the total mass distribution in dark matter haloes. Since there is not a single analytic model to describe halo density profiles across the whole range of halo masses we introduce two transition masses between dwarf to early-type galaxies and early-type to cluster of galaxies, respectively. For the description of early-type galaxy haloes we consider two approaches, the SIS and SISSA models, while for dwarfs and cluster of galaxies we adopt the NFW model. We utilize our samples of strong lenses from which we derive the observed distribution of image separation, in order to constrain the values of the transition masses. We were able to set good constrains on the maximum transition mass (see Table 3). Our results agree with previous studies of strong lens statistics using the CLASS (Browne et al. 2003; Myers et al. 2003) sample of strong lenses, where they place the value of the upper transition mass at $\sim 10^{13} \mathrm{M}_{\odot}$ (Porciani \& Madau 2000; Kochanek \& White 2001; Li \& Ostriker 2002; Oguri 2002; Kuhlen et al. 2004). A complementary approach was adopted by Oguri (2006) in which the author used a two-component halo density profile, composed of an NFW dark matter halo and a Hernquist model for the central galaxy, that also 


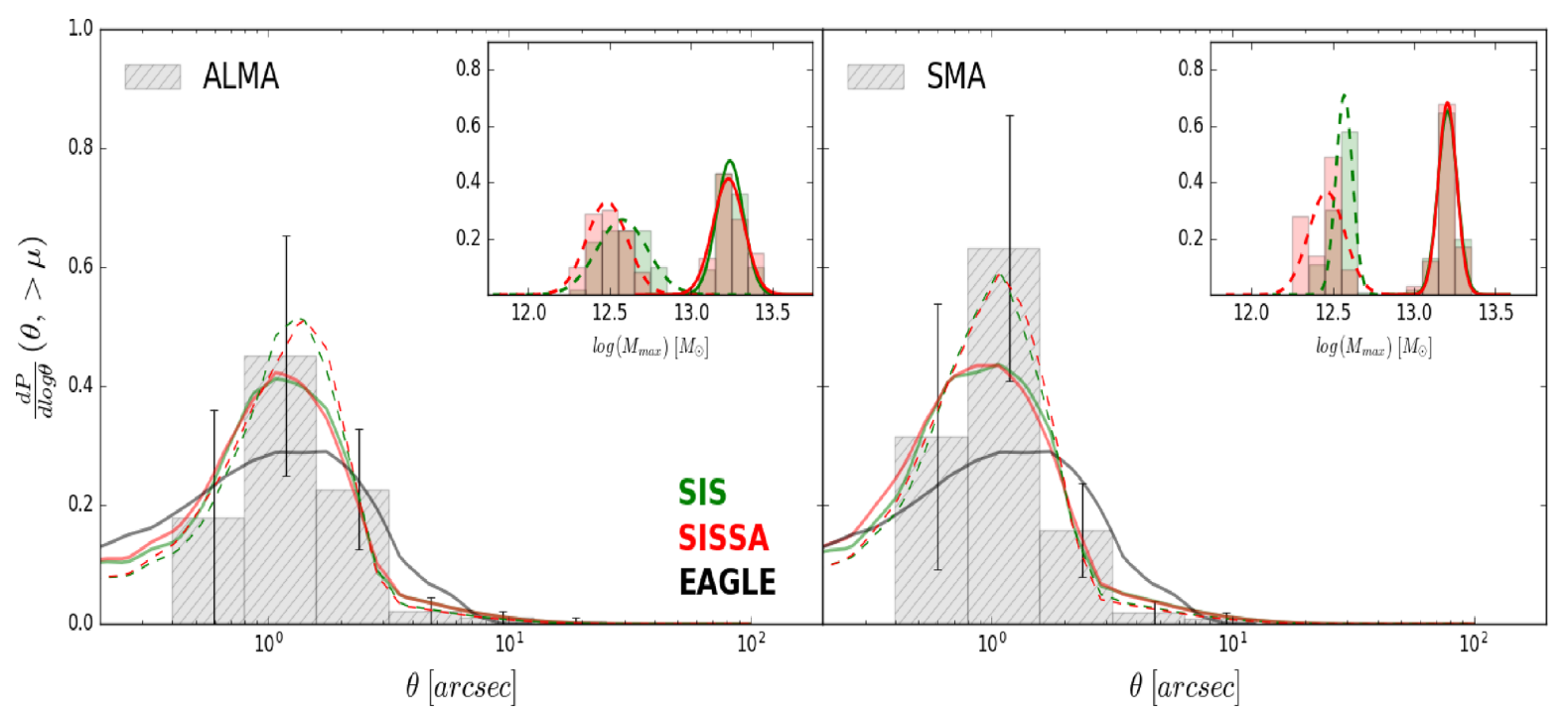

Figure 6. The predicted distribution of image separations adopting either the SIS (green) or SISSA (red) profiles for galaxy-scale lenses and following the procedure described in Section 4.1. The predicted distribution of image separation, which was derived assuming a halo model calibrated from the EAGLE simulation results, is shown with black dashed lines. Left- and right-hand panels correspond to the fits with the two samples of lenses followed-up with ALMA and SMA, respectively. The grey-scale histograms are the observed distributions of our samples. The figure insets show the distribution of the upper mass-transition parameter after performing $\sim 100$ realizations. The predictions adopting a virialization redshift $z_{1, \mathrm{v}}=z_{\mathrm{l}}$ are shown as straight lines, while the ones with a virialization redshift $z_{1, \mathrm{v}}=2.5$ are shown as dashed lines.

Table 3. Best-fitting value of the two transition masses that were used in our analytic model, adopting either the SIS or SISSA model for the description of galaxy-scale lenses. These values were derived assuming a virialization redshift $z_{1, \mathrm{v}}=z_{\mathrm{l}}$ for the first two rows and $z_{1, \mathrm{v}}=2.5$ for the last two.

\begin{tabular}{lcccc}
\hline & $\log \left(M_{\min }\right)_{\text {SIS }}$ & $\log \left(M_{\text {max }}\right)_{\text {SIS }}$ & $\log \left(M_{\min }\right)_{\text {SISSA }}$ & $\log \left(M_{\max }\right)_{\text {SISSA }}$ \\
\hline ALMA $_{z \text { vir }=z_{1}}$ & $\leq 12.4$ & $13.25 \pm 0.10$ & $\leq 12.3$ & $13.20 \pm 0.11$ \\
SMA $_{z \text { vir }}=z_{1}$ & $\leq 12.2$ & $13.19 \pm 0.07$ & $\leq 12.0$ & $13.20 \pm 0.06$ \\
ALMA $_{z \text { vir }}=2.5$ & $\leq 12.1$ & $12.56 \pm 0.13$ & $\leq 12.1$ & $12.48 \pm 0.10$ \\
SMA $_{\text {vir }=2.5}$ & $\leq 11.9$ & $12.54 \pm 0.07$ & $\leq 11.9$ & $12.42 \pm 0.10$ \\
\hline
\end{tabular}

considers the effect of adiabatic contraction of dark matter. This profile has a smooth transition between galaxy and cluster scale lenses and does not require the assumption of a transition mass and has the potential to better account for the contribution from groupscale lenses. This profile seem to provide a relatively good fit to radio (Oguri 2006) and optical data (More et al. 2012). However, as our sample is still limited in numbers to make such distinctions between models, we have not considered this approach.

A larger sample is also required in order to distinguish between models with a minimum transition mass $<10^{12} \mathrm{M}_{\odot}$ (Ma 2003). However, our candidate sample selection does not have any completeness issues at low angular resolutions as optical surveys do (More et al. 2016). This is because our selection is purely flux based and does not require the identification of individual multiply lensed images. Since our sample has no biases at small angular separation, follow up observations with ALMA can in fact probe the subarcsec scale of the image separation distribution (see e.g. HeLMS J235331.9+031718).

We also examined the effect of varying the virialization redshift of the lens $z_{1, v}$, which is one of the parameters of our analytic models. Previous studies of strong lens statistics have ignored its effect and always assumed that it coincides with the actual redshift of the halo $z_{1, \mathrm{v}}=z_{1}$. Lapi et al. (2012) argue that this approximation leads to an overestimate of the halo's size and, subsequently, to an underestimate of the lensing probability. We showed that adopting the value suggested by Lapi et al. (2012), $z_{1, \mathrm{v}}=2.5$, the constrained value of the maximum transition mass significantly decreases (see Table 3).

This approach of predicting the distribution of image separation based on the population of dark matter haloes selected on the basis of their halo mass provides a confirmation of the standard cold dark matter paradigm. However, the current samples of strong lenses are still not large enough in order to able to distinguish between the different models that attempt to describe the internal structure of these haloes. Scaling from the errors in Fig. 6 we estimate that a sample of $\sim 500$ would be required for this distinction to be made possible.

Is it practical to produce such a large sample of lensed sources. González-Nuevo et al. (2012) have proposed a method for finding at least 1000 lensed sources from the Herschel surveys. However, their method is based on finding galaxies that lie close to the position of a Herschel source, and therefore have a high probability of being associated with it, but which have much lower estimated redshifts than the Herschel source. This method will therefore be biased towards lensing systems with small image separations and so is not suitable for our purpose. 
The most promising method is a variant of the method used by Negrello et al. (2010). There are only $\simeq 150$ probable lensed sources with the 500- $\mu \mathrm{m}$ flux densities $>100 \mathrm{mJy}$ (Nayyeri et al. 2016; Negrello et al. 2017), the cut-off used by Negrello et al. (2010). However, Negrello et al. (2010) estimate that the fraction of highredshift Herschel sources that are strongly lensed is $>50$ percent down to a $500-\mu \mathrm{m}$ flux density of $\simeq 50 \mathrm{mJy}$. We have shown in this paper that observations with ALMA with exposure times of only a few minutes are enough to show that a bright Herschel source is lensed. Therefore, a programme to obtain short ALMA continuum observations of 500-1000 bright Herschel sources seems a practical way of assembling the required sample of 500 lensed systems. The more challenging part of the programme would be to obtain redshifts for the sources. However, 15-min ALMA observations are often enough to obtain a redshift for a bright Herschel source. Therefore, even this part of the project seems practical in an ALMA Large Program. In the slightly longer term, continuum surveys with the Square Kilometre Array will contain tens of thousands of lensed sources (Mancuso et al. 2015).

\section{ACKNOWLEDGEMENTS}

MN acknowledges financial support from the European Union's Horizon 2020 research and innovation programme under the Marie Skłodowska-Curie grant agreement no. 707601. Some of the spectroscopic redshift reported in this paper were obtained with the Southern African Large Telescope (SALT) under proposal 2015-2MLT-006, PI: Stephen Serjeant. LM acknowledges support from the South African Department of Science and Technology and the SA National Research Foundation. EV acknowledges funding from STFC consolidated grant ST/K000926/1. MWLS and SAE have received funding from the European Union Seventh Framework Programme ([FP7/2007-2013] [FP\&/2007-2011]) under grant agreement no. 607254. SJM and LD acknowledge support from the European Research Council (ERC) in the form of the Consolidator Grant COSMICDUST (ERC-2014-CoG-647939, PI: H. L. Gomez). SM, LD, and RJI acknowledge support from the ERC in the form of the Advanced Investigator Programme, COSMICISM (ERC-2012ADG_20120216, PI: R. J. Ivison). MJM acknowledges the support of the National Science Centre, Poland through the POLONEZ grant 2015/19/P/ST9/04010. This project has received funding from the European Union's Horizon 2020 research and innovation programme under the Marie Skłodowska-Curie grant agreement No. 665778

This paper makes use of the following ALMA data: ADS/JAO.ALMA\# 2013.1.00358.S. ALMA is a partnership of ESO (representing its member states), NSF (USA) and NINS (Japan), together with NRC (Canada), MOST and ASIAA (Taiwan), and KASI (Republic of Korea), in cooperation with the Republic of Chile. The Joint ALMA Observatory is operated by ESO, AUI/NRAO and NAOJ.

\section{REFERENCES}

Blain A. W., 1996, MNRAS, 283, 1340

Blumenthal G. R., Faber S. M., Flores R., Primack J. R., 1986, ApJ, 301, 27 Bocquet S., Saro A., Dolag K., Mohr J. J., 2016, MNRAS, 456, 2361

Bolton A. S., Burles S., Koopmans L. V. E., Treu T., Moustakas L. A., 2006, ApJ, 638, 703

Browne I. W. A. et al., 2003, MNRAS, 341, 13

Brownstein J. R. et al., 2012, ApJ, 744, 41
Bryan G. L., Norman M. L., 1998, ApJ, 495, 80

Bullock J. S., Kolatt T. S., Sigad Y., Somerville R. S., Kravtsov A. V., Klypin A. A., Primack J. R., Dekel A., 2001, MNRAS, 321, 559

Bussmann R. S. et al., 2013, ApJ, 779, 25

Calanog J. A. et al., 2014, ApJ, 797, 138

Chae K.-H., 2003, MNRAS, 346, 746

Cox P. et al., 2011, ApJ, 740, 63

Dye S. et al., 2014, MNRAS, 440, 2013

Dye S. et al., 2017, preprint (arXiv:1705.05413)

Eales S. A., 2015, MNRAS, 446, 3224

Eales S. et al., 2010, PASP, 122, 499

George R. D. et al., 2013, MNRAS, 436, L99

Gladders M. D., Yee H. K. C., 2005, ApJS, 157, 1

González-Nuevo J. et al., 2012, ApJ, 749, 65

Gruppioni C. et al., 2013, MNRAS, 432, 23

Harris A. I. et al., 2012, ApJ, 752, 152

Huterer D., Keeton C. R., Ma C.-P., 2005, ApJ, 624, 34

Inada N. et al., 2012, AJ, 143, 119

Jenkins A., Frenk C. S., White S. D. M., Colberg J. M., Cole S., Evrard A. E., Couchman H. M. P., Yoshida N., 2001, MNRAS, 321, 372

Kochanek C. S., White M., 2001, ApJ, 559, 531

Koopmans L. V. E., Treu T., Bolton A. S., Burles S., Moustakas L. A., 2006, ApJ, 649, 599

Koopmans L. V. E. et al., 2009, ApJ, 703, L51

Kuhlen M., Keeton C. R., Madau P., 2004, ApJ, 601, 104

Lapi A., Negrello M., González-Nuevo J., Cai Z.-Y., De Zotti G., Danese L., 2012, ApJ, 755, 46

Li L.-X., Ostriker J. P., 2002, ApJ, 566, 652

Li L.-X., Ostriker J. P., 2003, ApJ, 595, 603

Lupu R. E. et al., 2012, ApJ, 757, 135

Ma C.-P., 2003, ApJ, 584, L1

Mancuso C. et al., 2015, ApJ, 810, 72

Mason C. A. et al., 2015, ApJ, 805, 79

Messias H. et al., 2014, A\&A, 568, A92

More A., Cabanac R., More S., Alard C., Limousin M., Kneib J.-P., Gavazzi R., Motta V., 2012, ApJ, 749, 38

More A. et al., 2016, MNRAS, 455, 1191

Myers S. T. et al., 2003, MNRAS, 341, 1

Navarro J. F., Frenk C. S., White S. D. M., 1996, ApJ, 462, 563

Navarro J. F., Frenk C. S., White S. D. M., 1997, ApJ, 490, 493

Nayyeri H. et al., 2016, ApJ, 823, 17

Negrello M., Perrotta F., González-Nuevo J., Silva L., de Zotti G., Granato G. L., Baccigalupi C., Danese L., 2007, MNRAS, 377, 1557

Negrello M. et al., 2010, Science, 330, 800

Negrello M. et al., 2014, MNRAS, 440, 1999

Negrello M. et al., 2017, MNRAS, 465, 3558

Oguri M., 2002, ApJ, 580, 2

Oguri M., 2006, MNRAS, 367, 1241

Oguri M. et al., 2006, AJ, 132, 999

Oguri M. et al., 2008, AJ, 135, 512

Oguri M. et al., 2012, AJ, 143, 120

Oliver S. J. et al., 2012, MNRAS, 424, 1614

Omont A. et al., 2013, A\&A, 551, A115

Perrotta F., Baccigalupi C., Bartelmann M., De Zotti G., Granato G. L., 2002, MNRAS, 329, 445

Perrotta F., Magliocchetti M., Baccigalupi C., Bartelmann M., De Zotti G., Granato G. L., Silva L., Danese L., 2003, MNRAS, 338, 623

Pilbratt G. L. et al., 2010, A\&A, 518, L1

Planck Collaboration XVI, 2014, A\&A, 571, A16

Porciani C., Madau P., 2000, ApJ, 532, 679

Prada F., Klypin A. A., Cuesta A. J., Betancort-Rijo J. E., Primack J., 2012, MNRAS, 423, 3018

Prugniel P., Simien F., 1997, A\&A, 321, 111

Schaller M. et al., 2015a, MNRAS, 451, 1247

Schaller M. et al., 2015b, MNRAS, 452, 343

Schaye J. et al., 2015, MNRAS, 446, 521

Schneider P., Ehlers J., Falco E. E., 1992, Gravitational Lenses, XIV. Springer-Verlag, Berlin 
Sheth R. K., Tormen G., 1999, MNRAS, 308, 199

Stanford S. A., Gonzalez A. H., Brodwin M., Gettings D. P., Eisenhardt P. R. M., Stern D., Wylezalek D., 2014, ApJS, 213, 25

Takahashi R., Chiba T., 2001, ApJ, 563, 489

Tinker J., Kravtsov A. V., Klypin A., Abazajian K., Warren M. et al., 2008, ApJ, 688, 709 (T08)

Turner E. L., Ostriker J. P., Gott J. R., III, 1984, ApJ, 284, 1

Viero M. P. et al., 2014, ApJS, 210, 22

Wardlow J. L. et al., 2013, ApJ, 762, 59

White S. D. M., Rees M. J., 1978, MNRAS, 183, 341

\section{APPENDIX: UNDERSTANDING THE SISSA MODEL}

In this section we show the effects of the various ingredients that enter the calculation of cumulative image separation distribution (see Fig. A1). This is calculated from equation (25) by substituting the Dirac $\delta$ function by the Heaviside step function. For this particular calculation only we use the standard method for computing cross-sections as $\sigma=\pi \beta_{\mathrm{cr}}^{2}$, where $\beta_{\mathrm{cr}}$ is the radial caustic within which multiple images are formed.

\section{A1 Variation in $z_{\mathrm{s}}$}

The source redshift, $z_{\mathrm{s}}$, predominantly affects the amplitude of the distribution. This is to be expected since a higher source redshift corresponds to a larger volume of the Universe being considered. However, the predicted distributions of image separations in Section 4 are normalized and therefore this additional factor cancels out.

\section{A2 Variation in halo mass function}

The use of different halo mass functions models has very little effect on the distribution of image separations. The T08 and Bocquet mass functions assume the same formalism but their parameters are calibrated from dark matter only and Hydro cosmological simulations, respectively. We also make use of the halo mass function by Jenkins et al. (2001). Comparing the halo mass functions themselves we find that the effect of baryons is to suppress only slightly the creation of massive haloes but only at small redshifts. At higher redshifts they tend to agree fairly well.

\section{A3 Variation in $M_{\max }$}

The upper transition mass $M_{\max }$ parametrizes the change from galaxy-sized SISSA to group- and cluster-sized NFW lenses. This parameter determined the position of the kink in the image separation distribution. For an upper transition mass of $\log M_{\max }=13.50$ this transition occurs at $\theta=7$ arcsec. Lowering the transition mass to $\log M_{\max }=13.25$ shifts this transition down to $\theta=4 \operatorname{arcsec}$, while increasing it to $\log M_{\max }=13.75$ this transition shifts up to $\theta=10$ arcsec.

\section{A4 Variation in $M_{\text {vir }} / M_{\star}$ ratio}

The ratio between the halo and stellar mass, $M_{\mathrm{vir}} / M_{\star}$, is an important parameter in the SISSA model and its effect on the distribution of image separations is twofold. First, we see that increasing this ratio from 10 to 50, the abundance of arcsec-scale lenses decreases by almost a factor of $\sim 5$. Secondly, it affects the kink of the distribution by shifting it from $\theta=5$ to $10 \operatorname{arcsec}$.

\section{A5 Variation in $\sigma_{\log c}$}

The parameter $\sigma_{\log c}$ controls the standard deviation of the distribution of concentration parameters. This distribution is expected to have a scatter that is well described by a lognormal distribution:

$p(c)=\frac{1}{\sqrt{2 \pi} \sigma_{\log c} c} \exp \left[-\frac{(\log c-\log \bar{c})^{2}}{2 \sigma_{\log c}^{2}}\right]$,

where the $\bar{c}$ is given by equation (2). The SIS model does not depend on this parameter and therefore aresec-scale lenses produced by galaxies adopting this model are not affected by any changes (Takahashi \& Chiba 2001; Kuhlen et al. 2004; Oguri 2006). However, this parameter does enter in the SISSA model through the NFW component. Although, its effect is not as drastic as it is for the

\section{SISSA + NFW}

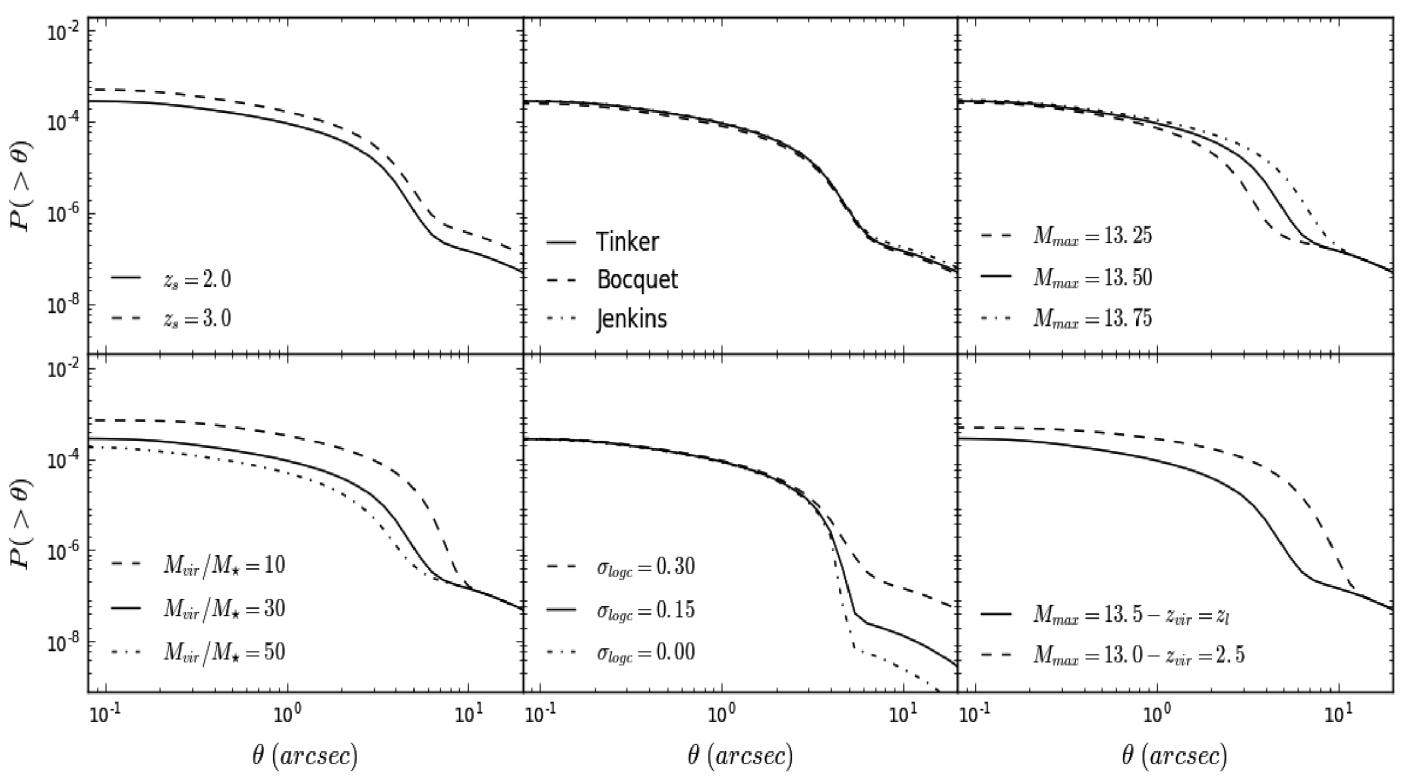

Figure A1. Effects of parameter variation in the cumulative distribution of image separations. 
wide-separation lenses produce by galaxy groups and cluster adopting a pure NFW model, it's still affects the resulting distribution of image separation by shifting the kink by a few arcsec.

\section{A6 Variation in $z_{\text {vir }}$}

As described in Section 3.1 the commonly made approximation that the virialization redshift in equal to the observed redshift lead to an overestimation of the halo size and therefore a decrease of the halo's density, making haloes less efficient. Adopting a virialization redshift $z_{1, \mathrm{v}}=2.5$ drastically shifts the kink of the distribution to larger angular scales, as well as it increases the abundance of galaxyscale lenses. In this case the virialization redshift is introduced only for the SISSA model, as it would be unrealistic to assume that group- and cluster-scale lenses had been virialiazed at such high redshift.

This paper has been typeset from a $\mathrm{T}_{\mathrm{E}} \mathrm{X} / \mathrm{LAT}_{\mathrm{E}} \mathrm{X}$ file prepared by the author. 